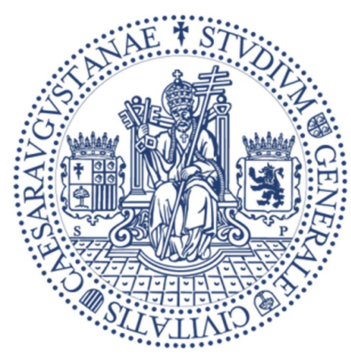

Universidad Zaragoza

\title{
Comentarios para la mejora en el posicionamiento de la UZ en algunos rankings universitarios
}

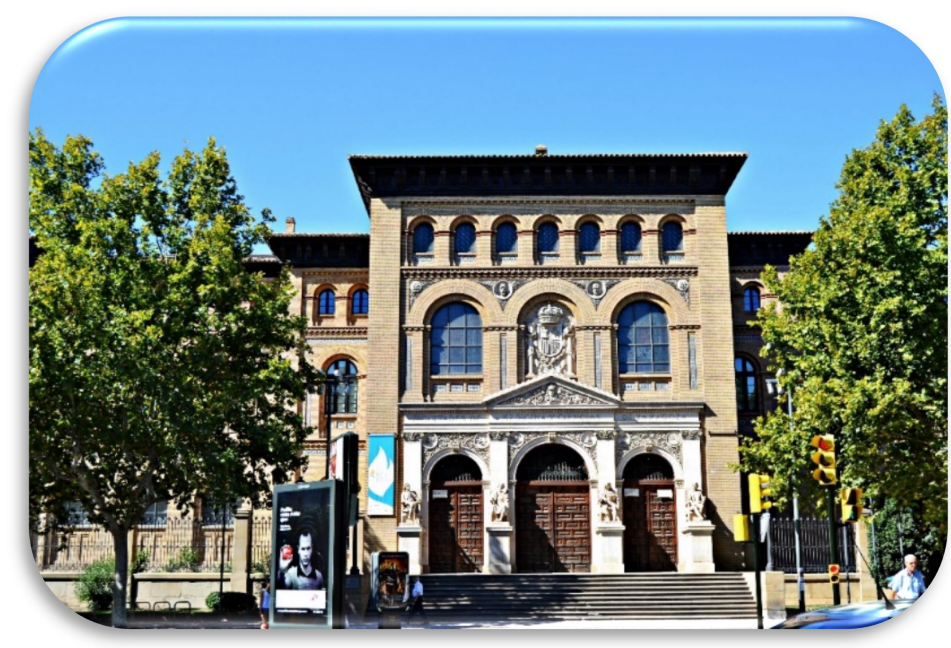

Informe generado por la Comisión de Rankings de la UZ

Francisco José Serón Arbeloa (Director) Manuel González Bedia (Secretario)

Juan Arana Pérez Celia Cañadas Blasco José María Gómez Sancho José Muñoz Embid Antonio Peiró Arroyo Agustín Urdangarín 
ISBN 978-84-16723-45-4

DOI 10.26754/posicion_UZ_rankings 


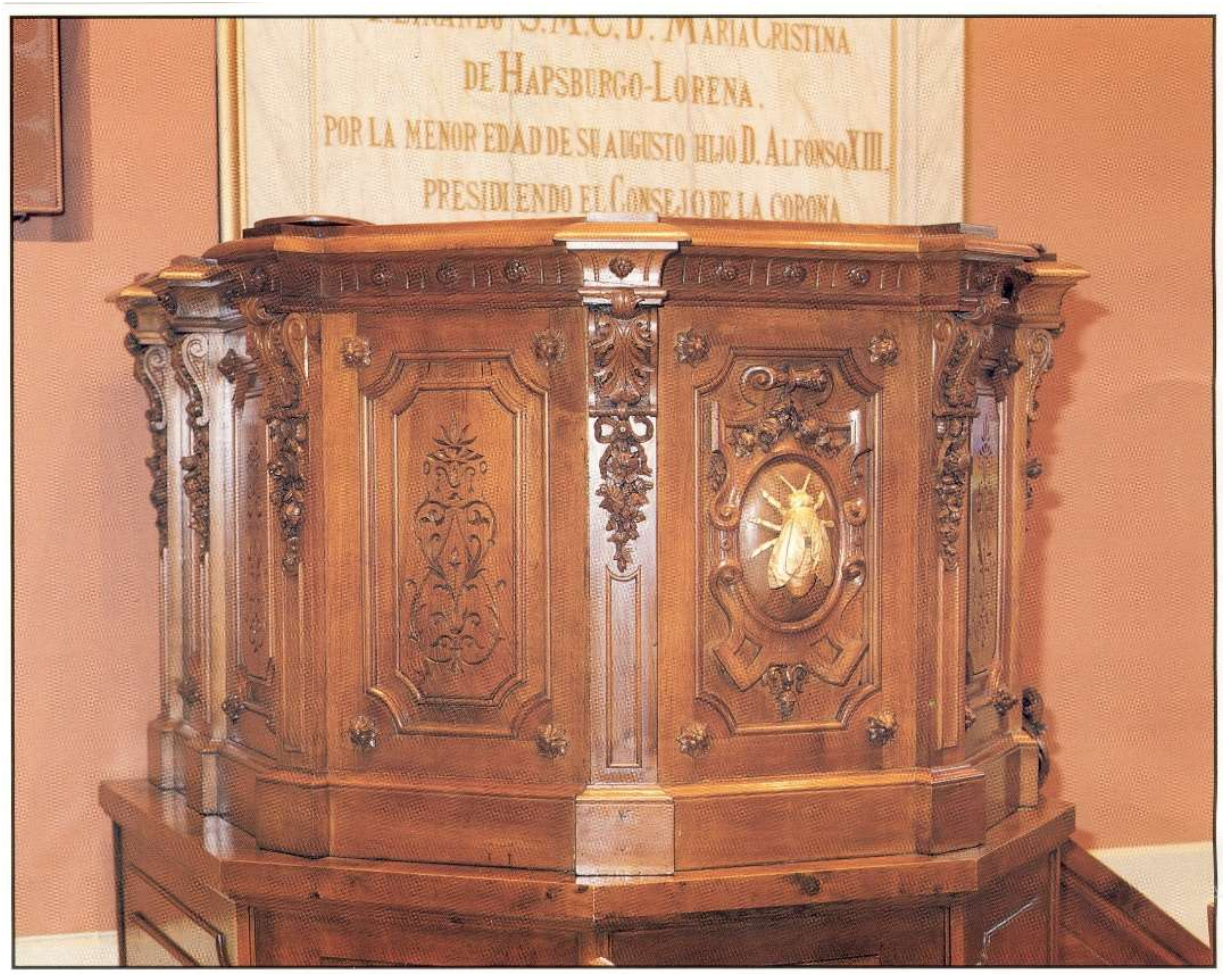

Cátedra del Paraninfo de la Universidad de Zaragoza

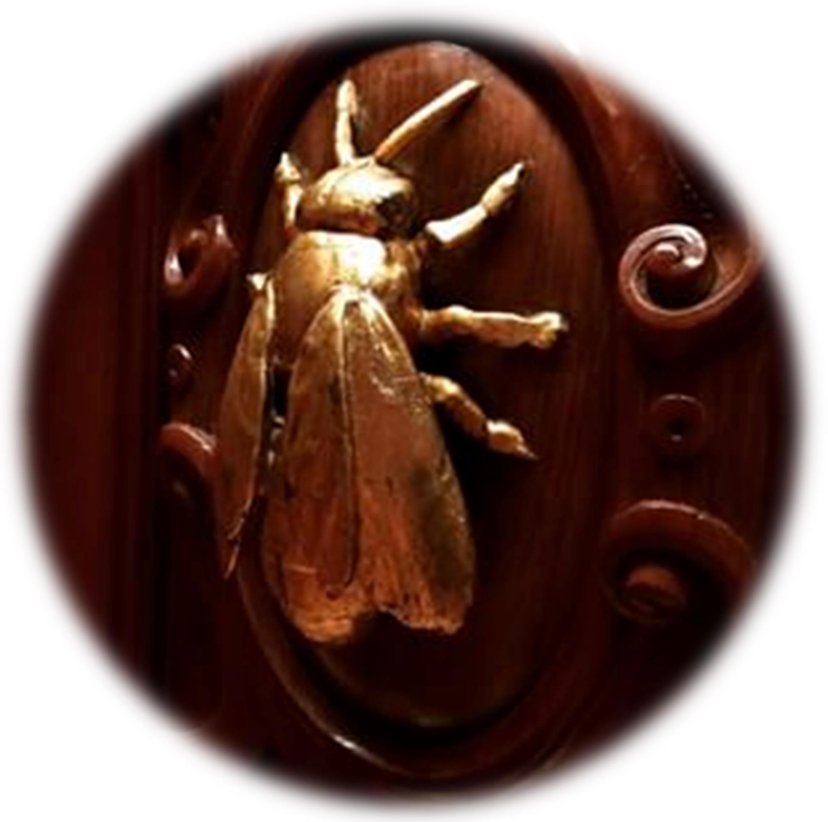

El púlpito o cátedra del Paraninfo de la Universidad de Zaragoza está en el lado de poniente, es tallado en madera y muestra, en relieve dorado, la figura de una abeja en su centro, como símbolo de la laboriosidad inteligente y ordenada y de la diligencia productiva. 
No hay dos hombres iguales

como no hay dos hojas iguales,

aunque pertenezcan a un mismo árbol

cada una toma el sol de una forma

$y$, sin embargo, todas sirven al árbol.

Cayetano Arroyo ("Yo soy tú mismo") 


\section{Índice}

- Introducción

06

- Rankings

○ Academic Ranking of World Universities (ARWU) 07

- Criterios de clasificación y ponderación 08

- Comentarios

09

○ Time Higher Education (THE)

- Criterios de clasificación y ponderación 12

- Comentarios

○ QS World Universitity Rankings

- Criterios de clasificación y ponderación 18

- Comentarios

- Apéndice 1

- Apéndice 2 


\section{Introducción}

Sin entrar en análisis críticos de oportunidad, planteamiento, o definición, aspectos que dependen tanto de los estamentos políticos como del propio profesorado, lo que es evidente es que los rankings universitarios se han consolidado como una forma simplista de evaluar la calidad de las instituciones universitarias. Tras las primeras clasificaciones, en las que las universidades se evaluaban globalmente, han ido apareciendo otras en las que se pormenorizan los resultados restringiendo el alcance de los grupos bajo análisis. Por ello, los grandes rankings comienzan a ofrecer clasificaciones equivalentes a lo que en España conocemos en el ámbito académico como macroáreas. Además, entre los factores que se tienen en cuenta, están los relativos a la calidad investigadora del profesorado, los relacionados con la docencia y otros de tipo social. Por tanto, es información cada vez más útil para darnos idea de cómo ven desde fuera a la Institución aunque sea de forma comparada con otras instituciones.

La Comisión de Rankings de la Universidad de Zaragoza se ha fijado en los tres Rankings generalistas más reconocidos y en cada caso, se recopila lo que tiene que ver con nuestra institución, se publican los resultados obtenidos, y en este informe se ofrecen sugerencias evidentes que se obtienen de los análisis que se han realizado.

Los rankings seleccionados son:

- Academic Ranking of World Universities (ARWU)

- $\quad$ Time Higher Education (THE)

- QS World Universitity Rankings

Los resultados aparecen en:

- $\quad$ https://www.unizar.es/rankings

Y los comentarios tan sólo pretenden mostrar aspectos que se han considerado de especial interés, resaltando aquellos pocos que podrían ser susceptibles de actuar sobre ellos, lo que permitiría mejorar nuestro posicionamiento en los próximos años.

Comisión de rankings de la $\mathrm{UZ}$ 


\section{Academic Ranking of World \\ Universities (ARWU) - Shangai}

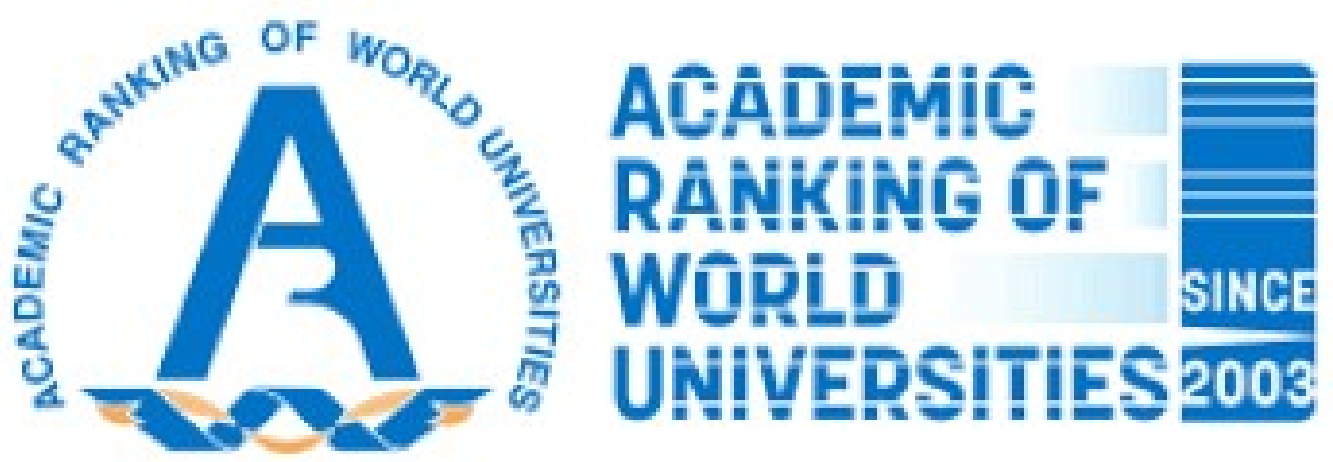

http://www.shanghairanking.com/ 


\section{Criterios de clasificación y ponderación}

Las universidades se clasifican según varios indicadores de rendimiento clasificables en dos tipos:

\section{Indicadores individuales}

- Alumni (10\%): número total de titulados que consiguieron ganar premios Nobel o medallas Fields.

- Award (20\%): número total de profesores de la institución que ganaron premios Nobel o medallas Fields.

- HiCi (20\%): número de investigadores altamente citados incluidos del listado HCR de Clarivate Analytics (www.highlycited.com).

\section{Indicadores colectivos}

- N\&S (20\%): número de artículos publicados en Science y Nature en los cinco años anteriores a la publicación del ranking.

- PUB (20\%): número de artículos indexados por el Science Citation Index Expanded y el Social Science Citation Index en el año anterior.

- PCP (10\%): Los valores de los cinco indicadores anteriores se agregan y dividen entre el número equivalente de personal académico permanente a tiempo completo. 


\section{Comentarios}

\section{Índices de difícil intervención o de modificación}

- Generar premios Nobel (indicador Alumni)

- Contratar premios Nobel (indicador Award)

\section{Índices de posible intervención}

- Contratación de investigadores HiCi. [Actualmente no tenemos ninguno]. Ver Apéndice 1. En él se muestra los valores de algunos de los indicadores que caracterizan a los únicos investigadores españoles que se consideran HiCi. La tabla está anonimizada.

- Programa de estímulo para que investigadores UZ envíen sus artículos a Nature \& Science.

- Aumento del número de publicaciones (indicador PUB), teniendo en cuenta además que las publicaciones indexadas el año anterior se organizan en tres grupos:

○ Las que están indexadas sólo en el Science Citation Index Expanded, que se valoran como una publicación.

- Las que están indexadas sólo en el Social Science Citation Index, que se valoran como dos publicaciones.

○ Las que aparecen en ambos índices, que se valoran como 1,5 publicaciónes.

Por lo tanto sería interesante, en el caso de que sea posible:

- Apostar por publicaciones interdisciplinares, ver Apéndice 2.

- Aumentar la producción en Ciencias Sociales y Humanidades en revistas indexadas. 


\section{THE (Times Higher Education) World University Rankings}
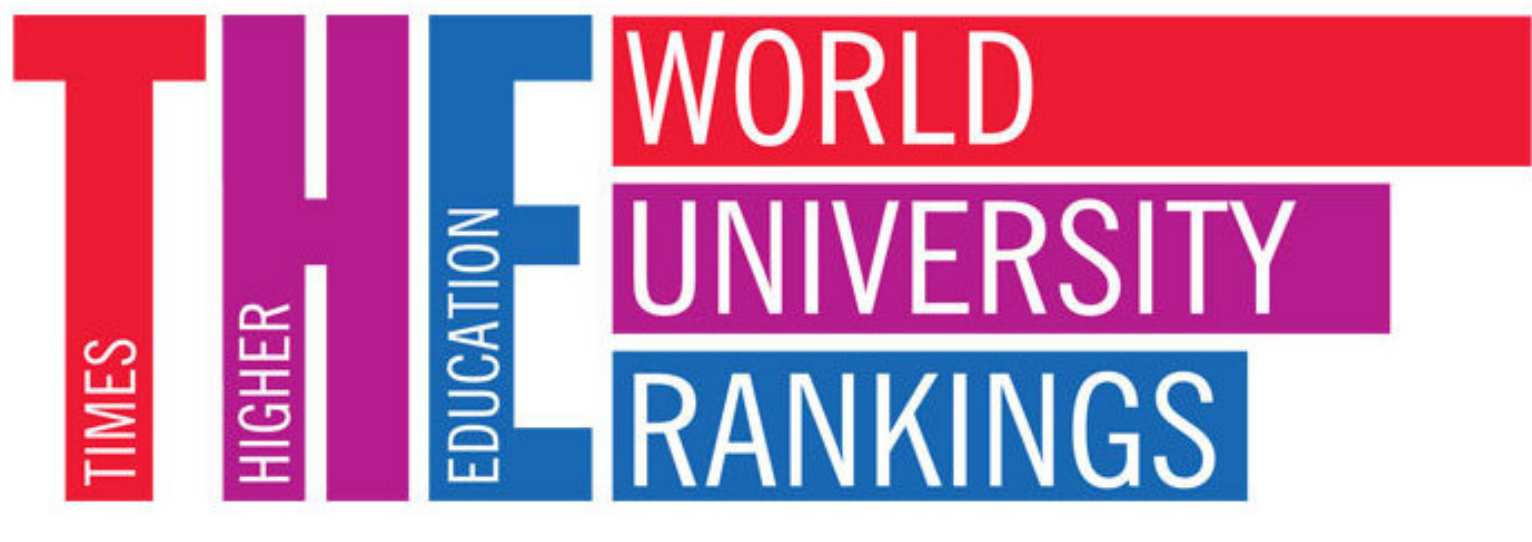

http://www.timeshighereducation.co.uk/ 


\section{Criterios de clasificación y ponderación}

El ranking del Times Higher Education juzga a las universidades en todas sus misiones principales: enseñanza, investigación, transferencia de conocimiento y proyección internacional. Utilizan 13 indicadores de rendimiento distribuidos en tales ámbitos.

1) TEACHING (30\%)
a) Reputation survey: $15 \%$
b) Staff-to-student ratio: $4.5 \%$
c) Doctorate-to-bachelor's ratio: $2.25 \%$
d) Doctorates-awarded- to-academic-staff ratio: $6 \%$
e) Institutional income: $2.25 \%$

La Encuesta de Reputación Académica se realiza anualmente y recoge el prestigio percibido de cada institución en docencia. La última en enero de 2017 obtuvo 10.568 respuestas y se combina con la de 2016 para trabajar con más de 20.000. El ingreso institucional se escala en función del personal académico y se normaliza.

\section{2) RESEARCH (30\%)}

a) Reputation survey: $18 \%$

b) Research income: $6 \%$

c) Research productivity: $6 \%$

El ingreso por investigación (controvertido) se escala en función del personal académico y se normaliza. Al normalizar se tiene en cuenta el perfil de cada institución ya que los ingresos en Ciencias o Ingenierías suelen ser más numerosos y mayores que los de Humanidades o Ciencias Sociales. Para medir la productividad de la investigación se cuenta el número de artículos publicados en las revistas académicas indexadas por la base de datos Scopus de Elsevier por profesor, escalado para el tamaño institucional y normalizado para el tema.

\section{3) CITAS (30\%)}

Se utiliza la Base de datos Scopus de Elsevier. En el último año, examinó casi 62 millones de citas a más de 12,4 millones de artículos de 23.000 revistas, revisiones de artículos, actas de conferencias, libros y capítulos de libros publicados durante los últimos cinco años. Los datos se normalizan para reflejar las variaciones en el volumen de citas entre diferentes áreas temáticas. En 2015-16, se excluyeron los artículos con más de 1,000 autores porque estaban teniendo un impacto desproporcionado en los puntajes de citas de un pequeño número de universidades. Desde el año pasado, se diseñó un método para reincorporar estos documentos. 


\section{4) PERSPECTIVA INTERNACIONAL (7,5\%)}

a) International-to-domestic-student ratio: $2.5 \%$

b) International-to-domestic-staff ratio: $2.5 \%$

c) International collaboration: $2.5 \%$

Para el indicador de colaboración internacional, se calcula la proporción de publicaciones que tienen al menos un coautor internacional y se premian participaciones más altos. Este indicador se normaliza para tener en cuenta la combinación de asignaturas de una universidad y utiliza la misma ventana de cinco años que la categoría que "Citas".

\section{5) INGRESOS INDUSTRIA (2,5\%)}

Esta categoría busca capturar la transferencia de conocimiento al observar cuánto ingreso de investigación obtiene una institución de la industria, en comparación con el número de personal académico que emplea.

\section{Caso particular: Ranking THE por áreas}

Para cada una de las 11 áreas que ellos han definido, varían los pesos de los indicadores (véase tabla siguiente) y los límites a partir de los cuáles se tienen en consideración la inclusión de una universidad en dichos rankings (número mínimo de publicaciones en los últimos 5 años en esa área y porcentaje mínimo de profesores trabajando en ella) 


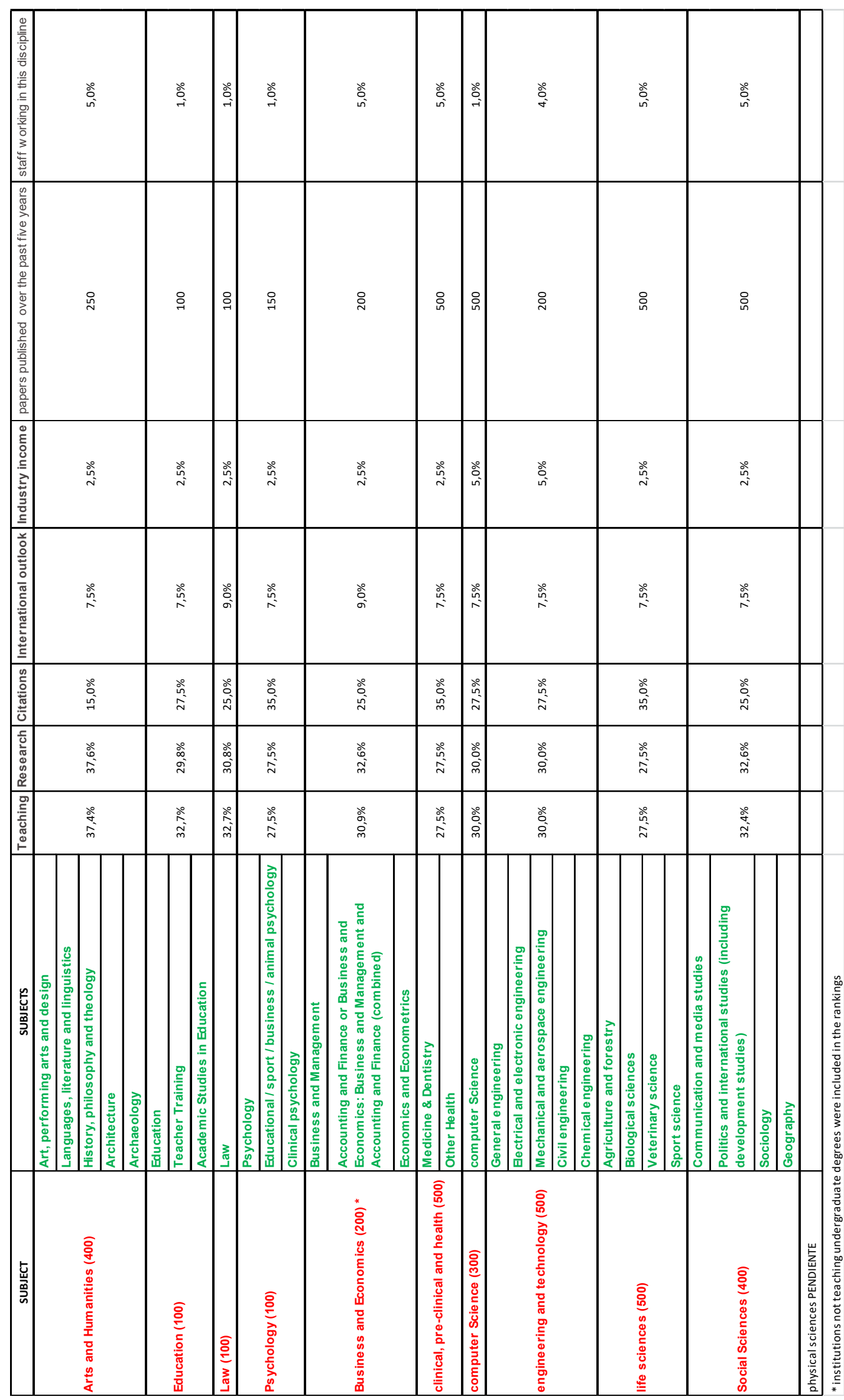




\section{Comentarios}

\section{Acciones de difícil intervención o de modificación}

La entrega de datos desde nuestra universidad, se proporciona agrupados en las grandes cinco macroáreas con las que se trabaja en España: humanidades (arts \& humanities), salud (clinical, preclinical \&health), ingenierías (engineering \& technology), ciencias (life sciences) y ciencias sociales (social sciences). Sin embargo es difícil entender cómo los usan para campos como: computer science, physical sciences, business \& economics, psychology, law, education.

\section{Índices de posible intervención}

A los ya indicadas en la página 7, podrían añadirse:

- En relación con la Encuesta de Reputación Académica (docencia e investigación): Se debería facilitar el acceso a la encuesta a estudiantes y profesorado.

- Mejorar la atracción de profesorado y alumnado extranjero. 


\section{QS World University Rankings}

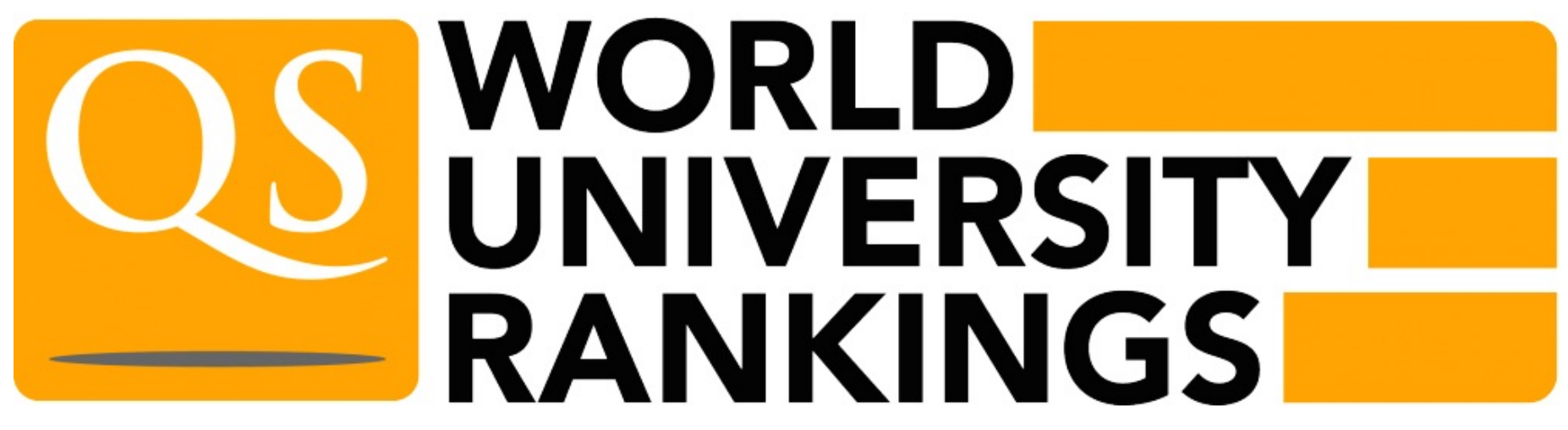

http://www.topuniversities.com/university-rankings/world-university-rankings/ 


\section{Criterios de clasificación y ponderación}

El ranking QS World University evalúa las Universidades de acuerdo con las siguientes seis métricas: Reputación académica, Reputación del empleador, Relación Facultad / Estudiante, Citas por área, Grado de internacionalización y Proporción de estudiantes internacionales.

1. Reputación académica (40\%): A través de una encuesta que recopila las opiniones de expertos de más de 70,000 personas en el ámbito de la educación superior con respecto a la calidad de la enseñanza y la investigación en las universidades del mundo.

2. Reputación del empleador (10\%): La métrica de Reputación del empleador se basa en más de 30,000 respuestas y le pide a los empleadores que identifiquen a aquellas instituciones de las cuales obtienen los graduados más competentes, innovadores y efectivos. Las respuestas nacionales e internacionales contribuirán en un 50\% cada una a la puntuación final de una institución.

3. Proporción docente / estudiante (20\%): La calidad de la enseñanza es típicamente citada por los estudiantes como la métrica de mayor importancia para ellos cuando se comparan las instituciones que usan una clasificación. Esta métrica evalúa la medida en que las instituciones pueden proporcionar a los estudiantes un acceso significativo a los profesores y tutores.

4. Citas por facultad (20\%): Se toma el número total de citas recibidas por todos los documentos producidos por una institución en un período de cinco años y se multiplica por el número de miembros de la facultad en esa institución. De esta manera se pretende tener en cuenta el hecho de que diferentes campos tienen culturas de publicación muy diferentes y se normaliza por las citas. Dado que las nuevas investigaciones requieren tiempo para ser diseminadas de manera efectiva en toda la comunidad académica, se toman las citas de los 5 años anteriores al que se evalua. Todos los datos de las citas se obtienen a partir de la base de datos Scopus de Elsevier.

5. Proporción de profesores internacionales y proporción de estudiantes internacionales (5\% cada uno). Una universidad altamente internacional adquiere y confiere una serie de ventajas. Inicialmente demuestra que tiene capacidad de atraer profesores y estudiantes de todo el mundo, lo que a su vez sugiere que posee una fuerte marca internacional. Ambas métricas valen el 5\% del total general. 


\section{Comentarios}

\section{Acciones de difícil intervención o de modificación}

- Academic reputation (40\%)

$\mathrm{Su}$ peso es enorme, pero es difícil actuar sobre este índice ya que los profesores/investigadores no pueden valorar a su propia institución.

\section{- Faculty/Student Ratio (20\%)}

Si se dispusiera de los medios, parece claro que la Universidad trabajaría para rebajar esta proporción por lo que no parece que sea un índice sobre el que se pueda actuar a corto plazo.

\section{- Citations per faculty $(20 \%)$}

Dado que se trata de citas globales y no de criterios de excelencia, es complicado actuar sobre este índice.

\section{Índices de posible intervención}

A los ya indicados en las página 7 y 13, podría añadirse:

\section{- Employer reputation (10\%)}

Índice sobre el que se podría incidir invitando a completar la encuesta correspondiente a empleadores.

\section{- International faculty ratio/International student ratio (5\% each)}

Podrían intervenirse para solucionar algunas dificultades administrativas en la gestión de solicitudes por parte de potenciales estudiantes extranjeros, como por ejemplo:

i) Facilitarles la atención personalizada fuera del horario laboral. Alto coste de las comunicaciones

ii) Facilitarles la obtención o recordatorio de credenciales para hacer trámites administrativos on-line (no suelen tener certificado electrónico ni conocidos en Aragón que les puedan hacer los trámites de identificación)

iii) Facilitarles información en inglés sobre procedimientos.

iv) Facilitarles los documentos que tienen que aportar (especialmente los que tienen que ver con, el reconocimiento de acreditaciones académicas o títulos extranjeros) 
Podrían analizarse algunas soluciones posibles:

a) Instalar sistemas de atención online basados en medios de comunicación populares: Whatsapp, Skype, Hangout. Fomentar horarios de atención fuera del horario laboral habitual.

b) Establecer sistemas de identificadores "ligeros" para realizar trámites de modo que no sea necesario tener certificado electrónico, desplazarse a registros la propia persona o algún representante.

c) Facilitar que cierta información esencial esté también en inglés (disponer de servicios de traducción sobre procedimientos y gestiones).

d) Facilitar la admisión de documentos acreditativos extranjero (títulos, etc.). 


\section{Apéndice 1}

En la siguiente tabla se muestran los valores de algunos de los indicadores que caracterizan a los únicos investigadores españoles que en este momento se consideran HiCi. La tabla está anonimizada.

En dicha tabla, la definición de los símbolos utilizados es la siguiente:

- H representa el índice $\mathrm{H}$

- TP número total de publicaciones

- $\mathrm{CP}$ citas promedio

- HCP publicaciones altamente citadas

- CsA citas sin autocitas

$\begin{array}{llllllll}\text { Afiliación } & \text { Materia } & \text { H } & \text { TP } & \text { CP } & \text { HCP } & \text { Citas } & \text { Cs.A }\end{array}$

\begin{tabular}{|c|c|c|c|c|c|c|c|}
\hline CSIC & Agricultural Sciences & 25 & 88 & 28,32 & 6 & 2494 & 2271 \\
\hline Univ Barcelona & Agricultural Sciences & 46 & 201 & 35,1 & 5 & 7055 & 6455 \\
\hline CREAF-CSIC & Agricultural Sciences & & & & 6 & & \\
\hline Hosp 12 Octubre & Agricultural Sciences & 43 & 91 & 77,48 & 5 & 7051 & 6671 \\
\hline Univ Barcelona & Agricultural Sciences & 69 & 244 & 59,2 & 13 & 14446 & 13396 \\
\hline Univ Jaume I & Chemistry & 67 & 264 & 60,3 & 33 & 15920 & 14938 \\
\hline CSIC & Chemistry & 68 & 461 & 40,28 & 35 & 18570 & 17346 \\
\hline Univ Politec Valencia & Chemistry & 59 & 360 & 38,68 & 25 & 13926 & 12499 \\
\hline Univ Illes Balears & Chemistry & 52 & 115 & 99,03 & 27 & 11389 & 10767 \\
\hline IDIBAPS & Clinical Medicine & 45 & 242 & 31,31 & 22 & 7577 & 7200 \\
\hline Univ Barcelona & Clinical Medicine & 51 & 202 & 118,18 & 29 & 23827 & 23209 \\
\hline Univ Barcelona & Clinical Medicine & 58 & 339 & 48,86 & 21 & 16562 & 15831 \\
\hline $\mathrm{CIC}$ biomaGUNE & Clinical Medicine & 59 & 207 & 114,68 & 45 & 23739 & 22996 \\
\hline $\begin{array}{l}\text { Univ Santiago de } \\
\text { Compostela }\end{array}$ & Clinical Medicine & 36 & 219 & 59,74 & 26 & 13082 & 12615 \\
\hline CSIC & Clinical Medicine & 54 & 461 & 39,64 & 44 & 18274 & 17972 \\
\hline CSIC & Clinical Medicine & 47 & 246 & 78 & 25 & 19224 & 19061 \\
\hline Univ Granada & Computer Science & 25 & 105 & 18,54 & 5 & 1947 & 1749 \\
\hline Univ Granada & Computer Science & 26 & 165 & 28,21 & 8 & 4655 & 4325 \\
\hline Univ Granada & Computer Science & 49 & 301 & 31,86 & 18 & 9591 & 8550 \\
\hline Univ Granada & Computer Science & 41 & 226 & 24,91 & 20 & 5629 & 4509 \\
\hline IDIBAPS & Computer Science & 22 & 110 & 32,79 & & 3607 & 3451 \\
\hline Univ Pompeu Fabra & Computer Science & 20 & 141 & 15,42 & 8 & 2174 & 1975 \\
\hline $\begin{array}{l}\text { Univ Miguel } \\
\text { Hernandez }\end{array}$ & Computer Science & 20 & 79 & 173,1 & & 13675 & 13597 \\
\hline CSIC & Engineering & 38 & 121 & 42,8 & 10 & 5179 & 4795 \\
\hline CSIC & Engineering & 45 & 193 & 38,89 & 8 & 7506 & 6256 \\
\hline CSIC & Engineering & 45 & 170 & 41,66 & 8 & 7082 & 5942 \\
\hline CSIC & Engineering & 45 & 169 & 41,78 & 8 & 7061 & 5919 \\
\hline
\end{tabular}




\begin{tabular}{|c|c|c|c|c|c|c|c|}
\hline CSIC & Engineering & 29 & 59 & 48,97 & 7 & 2889 & 2640 \\
\hline Univ Granada & Engineering & 49 & 301 & 31,86 & 16 & 9591 & 8550 \\
\hline Univ Granada & Engineering & 41 & 226 & 24,91 & 12 & 5629 & 4509 \\
\hline FIDMAG & Engineering & 29 & 203 & 20,05 & 10 & 4071 & 3960 \\
\hline CSIC & Environment/Ecology & 49 & 122 & 100,2 & 25 & 12,224 & 11667 \\
\hline ICREA & Environment/Ecology & 39 & 277 & 24,68 & 15 & 6835 & 6040 \\
\hline CSIC & Environment/Ecology & 35 & 150 & 36,04 & 11 & 5406 & 5103 \\
\hline Univ Barcelona & Environment/Ecology & 37 & 143 & 47,76 & 13 & 6829 & 6443 \\
\hline CIC biomaGUNE & Materials Science & 67 & 330 & 51,84 & 9 & 17107 & 15732 \\
\hline Univ Jaume I & Mathematics & & & & 29 & & \\
\hline Univ Loyola Andalucia & Mathematics & & & & 7 & & \\
\hline Ctr Genomic Reg & $\begin{array}{l}\text { Molecular Biology \& } \\
\text { Genetics }\end{array}$ & 55 & 138 & 160,91 & 17 & 22205 & 21918 \\
\hline IDIBAPS & $\begin{array}{l}\text { Neuroscience \& } \\
\text { Behavior }\end{array}$ & 44 & 221 & 32,79 & 20 & 7426 & 6489 \\
\hline Univ Barcelona & $\begin{array}{l}\text { Neuroscience \& } \\
\text { Behavior }\end{array}$ & 32 & 164 & 32,74 & 12 & 5369 & 5078 \\
\hline $\begin{array}{l}\text { Vall d'Hebron Inst } \\
\text { Oncol }\end{array}$ & $\begin{array}{l}\text { Neuroscience \& } \\
\text { Behavior }\end{array}$ & 47 & 157 & 43,58 & 12 & 6842 & 6649 \\
\hline Univ Basque Country & \begin{tabular}{|l|} 
Physics \\
\end{tabular} & 48 & 176 & 65,2 & 11 & 11475 & 10781 \\
\hline $\begin{array}{l}\text { Univ Autonoma } \\
\text { Madrid }\end{array}$ & Physics & 60 & 224 & 78,65 & 11 & 17618 & 16876 \\
\hline IMDEA Nanosci Inst & Physics & 53 & 149 & 148,87 & 29 & 22181 & 21675 \\
\hline $\begin{array}{l}\text { ICFO Inst Ciencies } \\
\text { Foton }\end{array}$ & Physics & 47 & 309 & 25,21 & 15 & 7791 & 7020 \\
\hline $\begin{array}{l}\text { Univ Santiago de } \\
\text { Compostela }\end{array}$ & Physics & 41 & 118 & 54,76 & 9 & 6462 & 6018 \\
\hline Univ Illes Balears & \begin{tabular}{|l|}
$\begin{array}{l}\text { Plant \& Animal } \\
\text { Science }\end{array}$ \\
\end{tabular} & 31 & 83 & 39,59 & 16 & 3286 & 2991 \\
\hline Univ Jaume I & $\begin{array}{l}\text { Plant \& Animal } \\
\text { Science }\end{array}$ & 22 & 43 & 49,07 & 10 & 2110 & 1985 \\
\hline Univ Jaen & $\begin{array}{l}\text { Plant \& Animal } \\
\text { Science }\end{array}$ & 24 & 70 & 33,11 & 11 & 2318 & 2082 \\
\hline CSIC & $\begin{array}{l}\text { Plant \& Animal } \\
\text { Science }\end{array}$ & 27 & 62 & 62,87 & 11 & 3898 & 3732 \\
\hline Univ Pompeu Fabra & Psychiatry/Psychology & 49 & 269 & 46,24 & 19 & 12438 & 12163 \\
\hline Univ Vigo & Psychiatry/Psychology & 28 & 96 & 30,41 & 13 & 2919 & 2715 \\
\hline$\overline{C S I C}$ & Psychiatry/Psychology & 56 & 395 & 26,96 & 17 & 10648 & 9108 \\
\hline
\end{tabular}




\section{Apéndice 2}

Número de revistas en Science Citation Index, Social Science Citation Index, y duplicadas entre ambos índices.

- 8.879 en Science

- 3.241 en Social Science

- 0.633 tanto en Science como en Social Science

Total de revistas diferentes considerando ambos repositorios $=11.487$

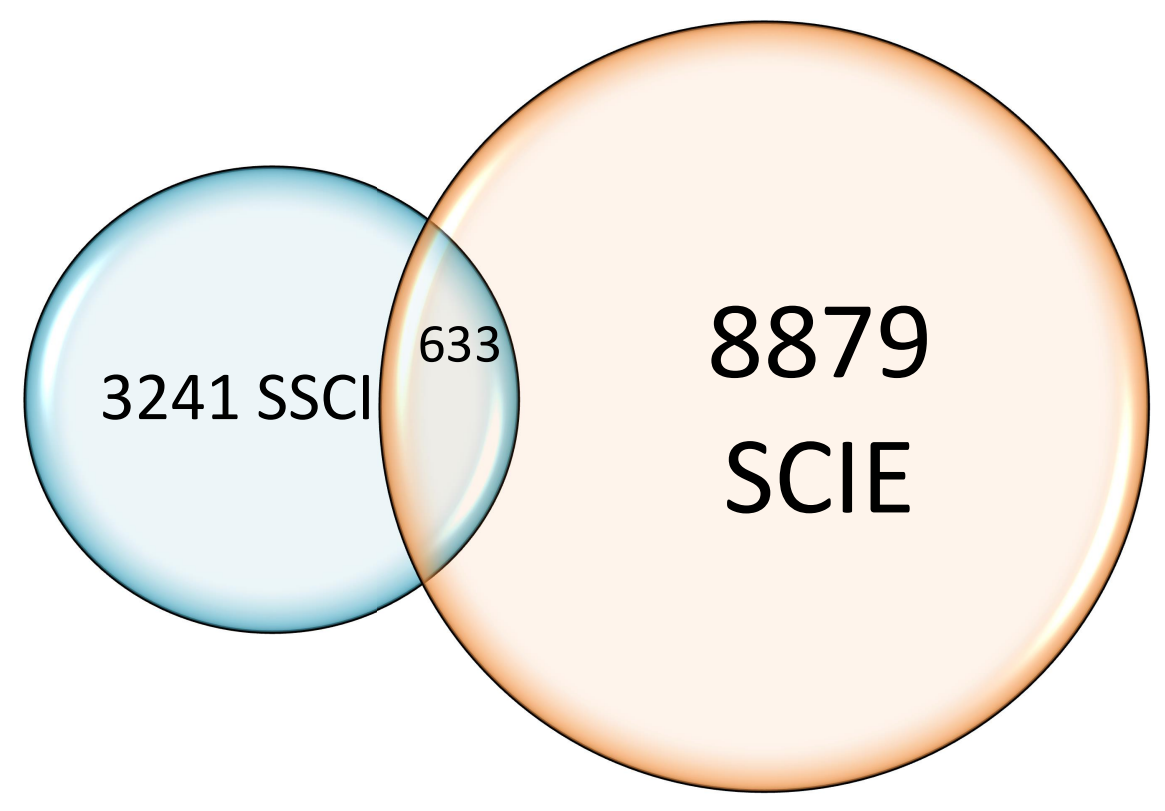

Si las clasificamos en función de la categoría que ocupan en WoS, las revistas aparecen en 233 categorías, en concreto:

- 177 categorías en Science.

- 056 categorías en Social Science.

A continuación mostraremos información tabuladas sobre las 633 revistas en dos tablas.

En la Tabla 1, se ordenan las categorías a las que pertenecen las revistas, tomando como criterio el número de revistas duplicadas. Campos como Nursing con más de 100 revistas duplicadas, Psychiatry con más de 80, o Public, Environmental \& Occupational Health con más de 70.

Solo se muestran las 50 categorías que tienen más del 10\%de sus revistas duplicadas en Science y Social Science.

En la Tabla 2 se muestra el listado de las 633 revistas duplicadas por orden alfabético. 
Tabla 1. 50 categorías que tienen más del 10\%de sus revistas duplicadas en Science y Social Science, ordenadas de mayor número de revistas duplicadas a menor.

\begin{tabular}{|c|c|c|c|c|c|}
\hline Category & & Edition & Journals & $\begin{array}{l}\text { revistas } \\
\text { duplicadas }\end{array}$ & $\begin{array}{c}\% \\
\text { duplicadas }\end{array}$ \\
\hline NURSING & 41 & SCIE & 116 & 112 & $96,55 \%$ \\
\hline NURSING & 13 & SSCI & 114 & 111 & $97,37 \%$ \\
\hline PSYCHIATRY & 26 & SCIE & 142 & 88 & $61,97 \%$ \\
\hline PSYCHIATRY & 9 & SSCI & 139 & 80 & $57,55 \%$ \\
\hline PUBLIC, ENVIRONMENTAL \& OCCUPATIONAL HEALTH & 6 & SSCI & 157 & 73 & $46,50 \%$ \\
\hline PUBLIC, ENVIRONMENTAL \& OCCUPATIONAL HEALTH & 15 & SCIE & 176 & 72 & $40,91 \%$ \\
\hline $\begin{array}{ll} & \text { PSYCHOLOGY } \\
\end{array}$ & 72 & SCIE & 77 & 60 & $77,92 \%$ \\
\hline HEALTH CARE SCIENCES \& SERVICES & 51 & SCIE & 90 & 49 & $54,44 \%$ \\
\hline ECONOMICS & 1 & SSCI & 347 & 44 & $12,68 \%$ \\
\hline HEALTH POLICY \& SERVICES & 25 & SSCI & 77 & 43 & $55,84 \%$ \\
\hline HISTORY \& PHILOSOPHY OF SCIENCE & 90 & SCIE & 60 & 39 & $65,00 \%$ \\
\hline HISTORY \& PHILOSOPHY OF SCIENCE & 37 & SSCI & 44 & 37 & $84,09 \%$ \\
\hline MATHEMATICS, INTERDISCIPLINARY APPLICATIONS & 46 & SCIE & 100 & 33 & $33,00 \%$ \\
\hline PSYCHOLOGY, EXPERIMENTAL & 20 & SSCI & 84 & 33 & $39,29 \%$ \\
\hline SOCIAL SCIENCES, MATHEMATICAL METHODS & 34 & SSCI & 49 & 33 & $67,35 \%$ \\
\hline MANAGEMENT & 3 & SSCI & 194 & 27 & $13,92 \%$ \\
\hline ENVIRONMENTAL STUDIES & 14 & SSCI & 105 & 26 & $24,76 \%$ \\
\hline PSYCHOLOGY, CLINICAL & 11 & SSCI & 121 & 25 & $20,66 \%$ \\
\hline REHABILITATION & 26 & SSCI & 70 & 25 & $35,71 \%$ \\
\hline REHABILITATION & 82 & SCIE & 65 & 23 & $35,38 \%$ \\
\hline INFORMATION SCIENCE \& LIBRARY SCIENCE & 19 & SSCI & 85 & 22 & $25,88 \%$ \\
\hline $\begin{array}{l}\text { STATISTICS \& PROBABILITY } \\
\end{array}$ & 39 & SCIE & 124 & 20 & $16,13 \%$ \\
\hline BEHAVIORAL SCIENCES & 105 & SCIE & 51 & 19 & $37,25 \%$ \\
\hline COMPUTER SCIENCE, INFORMATION SYSTEMS & 24 & SCIE & 146 & 19 & $13,01 \%$ \\
\hline NEUROSCIENCES & 5 & SCIE & 259 & 19 & $7,34 \%$ \\
\hline ENVIRONMENTAL SCIENCES & 8 & SCIE & 229 & 16 & $6,99 \%$ \\
\hline GERIATRICS \& GERONTOLOGY & 107 & SCIE & 49 & 16 & $32,65 \%$ \\
\hline GERONTOLOGY & 49 & SSCI & 32 & 16 & $50,00 \%$ \\
\hline $\begin{array}{ll}\text { LINGUISTICS } \\
\end{array}$ & 4 & SSCI & 182 & 16 & $8,79 \%$ \\
\hline OPERATIONS RESEARCH \& MANAGEMENT SCIENCE & 63 & SCIE & 83 & 16 & $19,28 \%$ \\
\hline PSYCHOLOGY, MULTIDISCIPLINARY & 10 & SSCI & 129 & 16 & $12,40 \%$ \\
\hline SPORT SCIENCES & 65 & SCIE & 81 & 16 & $19,75 \%$ \\
\hline EDUCATION \& EDUCATIONAL RESEARCH & 2 & SSCI & 235 & 15 & $6,38 \%$ \\
\hline $\begin{array}{r}\text { ETHICS } \\
\end{array}$ & 33 & SSCI & 51 & 15 & $29,41 \%$ \\
\hline SOCIAL SCIENCES, BIOMEDICAL & 42 & SSCI & 40 & 15 & $37,50 \%$ \\
\hline AGRICULTURAL ECONOMICS \& POLICY & 169 & SCIE & 17 & 14 & $82,35 \%$ \\
\hline AUDIOLOGY \& SPEECH-LANGUAGE PATHOLOGY & 149 & SCIE & 25 & 14 & $56,00 \%$ \\
\hline ENGINEERING, INDUSTRIAL & 113 & SCIE & 44 & 14 & $31,82 \%$ \\
\hline PEDIATRICS & 40 & SCIE & 121 & 14 & $11,57 \%$ \\
\hline SUBSTANCE ABUSE & 168 & SCIE & 18 & 14 & $77,78 \%$ \\
\hline MEDICAL ETHICS & 170 & SCIE & 16 & 13 & $81,25 \%$ \\
\hline SUBSTANCE ABUSE & 47 & SSCI & 34 & 13 & $38,24 \%$ \\
\hline ERGONOMICS & 52 & SSCI & 16 & 12 & $75,00 \%$ \\
\hline PSYCHOLOGY, BIOLOGICAL & 54 & SSCI & 14 & 12 & $85,71 \%$ \\
\hline EDUCATION, SCIENTIFIC DISCIPLINES & 117 & SCIE & 41 & 11 & $26,83 \%$ \\
\hline COMPUTER SCIENCE, INTERDISCIPLINARY APPLICATIONS & 43 & SCIE & 105 & 10 & $9,52 \%$ \\
\hline PSYCHOLOGY, APPLIED & 22 & SSCI & 80 & 10 & $12,50 \%$ \\
\hline PSYCHOLOGY, DEVELOPMENTAL & 26 & SSCI & 70 & 10 & $14,29 \%$ \\
\hline TRANSPORTATION & 48 & SSCI & 33 & 10 & $30,30 \%$ \\
\hline TRANSPORTATION SCIENCE \& TECHNOLOGY & 123 & SCIE & 34 & 10 & $29,41 \%$ \\
\hline
\end{tabular}


Tabla 2. Listado de las 633 revistas duplicadas en SCI y SSCI ordenadas por orden alfabético

\begin{tabular}{|c|}
\hline Aorn Journal \\
\hline Acta Psychiatrica Scandinavica \\
\hline Behavior Genetics \\
\hline Journal Of Nursing Administration \\
\hline Agricultural History \\
\hline Journal Of The American Geriatrics Society \\
\hline American Journal Of Agricultural Economics \\
\hline American Journal Of Nursing \\
\hline American Journal Of Physical Anthropology \\
\hline American Journal Of Psychiatry \\
\hline Annals Of Science \\
\hline Applied Ergonomics \\
\hline Archive For History Of Exact Sciences \\
\hline Australian And New Zealand Journal Of Psychiatry \\
\hline British Journal For The Philosophy Of Science \\
\hline British Journal Of Mathematical \& Statistical Psychology \\
\hline British Journal Of Psychiatry \\
\hline Bulletin Of The History Of Medicine \\
\hline Canadian Journal Of Agricultural Economics-Revue Canadienne D Agroeconomie \\
\hline Canadian Journal Of Occupational Therapy-Revue Canadienne D Ergotherapie \\
\hline Centaurus \\
\hline Cognitive Psychology \\
\hline Comprehensive Psychiatry \\
\hline Econometrica \\
\hline Educational And Psychological Measurement \\
\hline Engineering Economist \\
\hline Environment \\
\hline Erde \\
\hline Erdkunde \\
\hline Ergonomics \\
\hline Health Services Research \\
\hline Human Biology \\
\hline Human Factors \\
\hline leee Transactions On Engineering Management \\
\hline Indian Journal Of Psychiatry \\
\hline International Journal Of Game Theory \\
\hline International Journal Of Health Services \\
\hline International Journal Of Nursing Studies \\
\hline International Nursing Review \\
\hline Isis \\
\hline Journal Of Agricultural Economics \\
\hline Journal Of Child Psychology And Psychiatry \\
\hline Journal Of Communication Disorders \\
\hline
\end{tabular}




\begin{tabular}{|c|}
\hline Journal Of Continuing Education In Nursing \\
\hline Journal Of Genetic Psychology \\
\hline Journal Of Mathematical Psychology \\
\hline Journal Of Mathematical Sociology \\
\hline Journal Of Motor Behavior \\
\hline Journal Of Nervous And Mental Disease \\
\hline Journal Of Psychiatric Research \\
\hline Journal Of Psychosomatic Research \\
\hline Journal Of School Health \\
\hline Journal Of The Experimental Analysis Of Behavior \\
\hline Journal Of The History Of Biology \\
\hline Journal Of The History Of Medicine And Allied Sciences \\
\hline Language And Speech \\
\hline Management Science \\
\hline Medical Care \\
\hline Medical History \\
\hline Medicine Science And The Law \\
\hline Multivariate Behavioral Research \\
\hline Neuropsychologia \\
\hline Nursing Clinics Of North America \\
\hline Nursing Outlook \\
\hline Nursing Research \\
\hline Operations Research \\
\hline Perspectives In Psychiatric Care \\
\hline Philosophy Of Science \\
\hline Phonetica \\
\hline Physiology \& Behavior \\
\hline Program-Electronic Library And Information Systems \\
\hline Psychiatry-Interpersonal And Biological Processes \\
\hline Psychological Bulletin \\
\hline Psychological Medicine \\
\hline Psychological Review \\
\hline Psychometrika \\
\hline Psychosomatic Medicine \\
\hline Psychosomatics \\
\hline Psychotherapy And Psychosomatics \\
\hline Public Health \\
\hline Public Health Reports \\
\hline Quality \& Quantity \\
\hline Quest \\
\hline Revista De Saude Publica \\
\hline Rivista Di Psichiatria \\
\hline Socio-Economic Planning Sciences \\
\hline Studies In History And Philosophy Of Science \\
\hline Synthese \\
\hline
\end{tabular}




\begin{tabular}{|c|}
\hline Technology And Culture \\
\hline Transportation Science \\
\hline Travail Humain \\
\hline Inquiry-The Journal Of Health Care Organization Provision And Financing \\
\hline International Journal Of Sport Psychology \\
\hline Journal Of Human Evolution \\
\hline Psychophysiology \\
\hline Transportation \\
\hline Annual Review Of Psychology \\
\hline History Of Science \\
\hline Psychiatrie De L Enfant \\
\hline Revista Da Escola De Enfermagem Da USP \\
\hline American Journal Of Public Health \\
\hline International Journal Of Psychiatry In Medicine \\
\hline Interfaces \\
\hline Hastings Center Report \\
\hline Brain And Language \\
\hline Journal Of Fluency Disorders \\
\hline American Journal Of Drug And Alcohol Abuse \\
\hline Aggressive Behavior \\
\hline Journal Of Experimental Psychology-Human Perception And Performance \\
\hline Journal Of Experimental Psychology-Animal Learning And Cognition \\
\hline Journal Of Gerontological Nursing \\
\hline Journal Of Emergency Nursing \\
\hline Cadernos De Saude Publica \\
\hline Acta Paulista De Enfermagem \\
\hline Scientometrics \\
\hline Agricultural Economics-Zemedelska Ekonomika \\
\hline Behavioral And Brain Sciences \\
\hline Journal Of Epidemiology And Community Health \\
\hline Behaviour \& Information Technology \\
\hline Diabetes Educator \\
\hline Journal Of Nursing Education \\
\hline Australian Health Review \\
\hline Journal Of The Operational Research Society \\
\hline Journal Of Clinical Psychiatry \\
\hline Research In Nursing \& Health \\
\hline Language Speech And Hearing Services In Schools \\
\hline Issues In Mental Health Nursing \\
\hline Advances In Nursing Science \\
\hline Cancer Nursing \\
\hline Evaluation \& The Health Professions \\
\hline Annual Review Of Public Health \\
\hline General Hospital Psychiatry \\
\hline Natural Resources Forum \\
\hline
\end{tabular}




\begin{tabular}{|c|}
\hline Journal Of Affective Disorders \\
\hline European Review Of Agricultural Economics \\
\hline Psychiatry Research \\
\hline Mathematical Social Sciences \\
\hline Journal Of Information Science \\
\hline Technovation \\
\hline Journal Of Psychosomatic Obstetrics \& Gynecology \\
\hline Journal Of Health Economics \\
\hline Insurance Mathematics \& Economics \\
\hline International Journal Of Psychophysiology \\
\hline Human Movement Science \\
\hline Health Policy \\
\hline Landscape And Urban Planning \\
\hline Biology \& Philosophy \\
\hline Agricultural Economics \\
\hline International Journal Of Industrial Ergonomics \\
\hline Berichte Zur Wissenschaftsgeschichte \\
\hline Journal Of Classification \\
\hline Oncology Nursing Forum \\
\hline Transportation Research Part B-Methodological \\
\hline Western Journal Of Nursing Research \\
\hline Physical \& Occupational Therapy In Pediatrics \\
\hline Energy Journal \\
\hline Journal Of Developmental And Behavioral Pediatrics \\
\hline Geriatric Nursing \\
\hline Journal Of Public Health Policy \\
\hline Dynamis \\
\hline Anales De Psicologia \\
\hline Gaceta Sanitaria \\
\hline Adicciones \\
\hline Psychopathology \\
\hline Nurse Education Today \\
\hline Archives Of Natural History \\
\hline Cognitive Neuropsychology \\
\hline Econometric Theory \\
\hline Midwifery \\
\hline International Journal Of Technology Management \\
\hline Health Policy And Planning \\
\hline Journal Of Information Technology \\
\hline Journal Of Psychophysiology \\
\hline Science In Context \\
\hline Brain Injury \\
\hline Clinical Linguistics \& Phonetics \\
\hline Bioethics \\
\hline Research Quarterly For Exercise And Sport \\
\hline
\end{tabular}




\begin{tabular}{|c|}
\hline Risk Analysis \\
\hline Journal Of Operations Management \\
\hline Journal Of Teaching In Physical Education \\
\hline International Journal Of Eating Disorders \\
\hline Mis Quarterly \\
\hline Social Science \& Medicine \\
\hline Journal Of Ethnobiology \\
\hline Brain And Cognition \\
\hline Health Affairs \\
\hline Rehabilitation Nursing \\
\hline Health Psychology \\
\hline Journal Of Experimental Psychology-Learning Memory And Cognition \\
\hline Journal Of Psychosocial Nursing And Mental Health Services \\
\hline Critical Care Nurse \\
\hline Journal Of Official Statistics \\
\hline Perception \\
\hline Biological Psychology \\
\hline Energy Policy \\
\hline Annals Of Human Biology \\
\hline Journal Of Mathematical Economics \\
\hline Journal Of Econometrics \\
\hline Omega-International Journal Of Management Science \\
\hline Child Care Health And Development \\
\hline Journal Of Archaeological Science \\
\hline Studies In Science Education \\
\hline Oxford Bulletin Of Economics And Statistics \\
\hline Social Studies Of Science \\
\hline Information Processing \& Management \\
\hline Addictive Behaviors \\
\hline Journal Of Medical Ethics \\
\hline Food Policy \\
\hline Interdisciplinary Science Reviews \\
\hline British Journal Of Occupational Therapy \\
\hline Telecommunications Policy \\
\hline Journal Of Advanced Nursing \\
\hline Historia Mathematica \\
\hline Revista Argentina De Clinica Psicologica \\
\hline Israel Journal Of Psychiatry And Related Sciences \\
\hline International Journal Of Rehabilitation Research \\
\hline Scandinavian Actuarial Journal \\
\hline Psychiatria Danubina \\
\hline Scandinavian Journal Of Work Environment \& Health \\
\hline Computers \& Education \\
\hline Journal Of Health Politics Policy And Law \\
\hline MCN-The American Journal Of Maternal-Child Nursing \\
\hline
\end{tabular}




\begin{tabular}{|c|}
\hline Nurse Educator \\
\hline Osiris \\
\hline Behavioural Processes \\
\hline Drug And Alcohol Dependence \\
\hline Information \& Management \\
\hline History And Philosophy Of The Life Sciences \\
\hline Nuncius-Journal Of The History Of Science \\
\hline Economic Computation And Economic Cybernetics Studies And Research \\
\hline Astin Bulletin \\
\hline Schizophrenia Bulletin \\
\hline Canadian Journal Of Psychiatry-Revue Canadienne De Psychiatrie \\
\hline Survey Methodology \\
\hline Revista De Geografia Norte Grande \\
\hline Recht \& Psychiatrie \\
\hline Birth-Issues In Perinatal Care \\
\hline Information Technology And Libraries \\
\hline Clinical Gerontologist \\
\hline Journal Of Urban Planning And Development \\
\hline Journal Of Business \& Economic Statistics \\
\hline Alcohol And Alcoholism \\
\hline Journal Of Comparative Psychology \\
\hline Earth Sciences History \\
\hline Journal Of Community Health Nursing \\
\hline Public Health Nursing \\
\hline Journal Of Product Innovation Management \\
\hline Marine Resource Economics \\
\hline Patient Education And Counseling \\
\hline Sociology Of Sport Journal \\
\hline Journal Of Management Information Systems \\
\hline Agribusiness \\
\hline Augmentative And Alternative Communication \\
\hline Orthopaedic Nursing \\
\hline Nursing Economics \\
\hline Econometric Reviews \\
\hline Issues In Science And Technology \\
\hline Journal Of Rehabilitation Research And Development \\
\hline Journal Of Memory And Language \\
\hline Nordic Journal Of Psychiatry \\
\hline Australian Journal Of Advanced Nursing \\
\hline Journal Of Palliative Care \\
\hline Health Reports \\
\hline Journal Of Pediatric Nursing-Nursing Care Of Children \& Families \\
\hline Archives Of Psychiatric Nursing \\
\hline Jognn-Journal Of Obstetric Gynecologic And Neonatal Nursing \\
\hline International Journal Of Geriatric Psychiatry \\
\hline
\end{tabular}




\begin{tabular}{|c|}
\hline Journal Of Head Trauma Rehabilitation \\
\hline Milbank Quarterly \\
\hline Archives Of Clinical Neuropsychology \\
\hline Clinical Nurse Specialist \\
\hline Holistic Nursing Practice \\
\hline Journal Of Neuroscience Nursing \\
\hline Journal Of Sport Management \\
\hline Sport Psychologist \\
\hline Agriculture And Human Values \\
\hline Journal Of Cardiovascular Nursing \\
\hline Substance Abuse \\
\hline Mathematical Population Studies \\
\hline Scientist \\
\hline Journal Of Rural Health \\
\hline Journal Of The American Academy Of Child And Adolescent Psychiatry \\
\hline Computational Linguistics \\
\hline Journal Of Pediatric Health Care \\
\hline Coastal Management \\
\hline Anthrozoos \\
\hline Journal Of Perinatal \& Neonatal Nursing \\
\hline Nursing Science Quarterly \\
\hline Neuropsychology \\
\hline Social Science Computer Review \\
\hline Journal Of Sport \& Exercise Psychology \\
\hline Research-Technology Management \\
\hline Behavioral Medicine \\
\hline Applied Nursing Research \\
\hline Journal Of Cognitive Neuroscience \\
\hline Critical Care Nursing Clinics Of North America \\
\hline Journal Of Neurolinguistics \\
\hline Schizophrenia Research \\
\hline Ecological Economics \\
\hline Journal Of Engineering And Technology Management \\
\hline European Psychiatry \\
\hline Science \& Education \\
\hline Computational Economics \\
\hline Social Psychiatry And Psychiatric Epidemiology \\
\hline GAIA-Ecological Perspectives For Science And Society \\
\hline Zeitschrift Fur Gerontologie Und Geriatrie \\
\hline Finance And Stochastics \\
\hline Social History Of Medicine \\
\hline Current Opinion In Psychiatry \\
\hline History Of The Human Sciences \\
\hline Interacting With Computers \\
\hline Utilities Policy \\
\hline
\end{tabular}




\begin{tabular}{|c|}
\hline Global Environmental Change-Human And Policy Dimensions \\
\hline European Journal Of Information Systems \\
\hline Mathematical Finance \\
\hline European Journal Of Cancer Care \\
\hline Journal Of Clinical Nursing \\
\hline Quality Of Life Research \\
\hline Cambridge Quarterly Of Healthcare Ethics \\
\hline Disability And Rehabilitation \\
\hline Journal Of Strategic Information Systems \\
\hline Journal Of The Royal Statistical Society Series A-Statistics In Society \\
\hline Intensive And Critical Care Nursing \\
\hline Tobacco Control \\
\hline Journal Of Tissue Viability \\
\hline Addiction \\
\hline Transportation Research Part A-Policy And Practice \\
\hline Journal Of Nursing Management \\
\hline Occupational Therapy International \\
\hline Medical Law Review \\
\hline International Transactions In Operational Research \\
\hline Nursing Ethics \\
\hline Asia-Pacific Journal Of Public Health \\
\hline Pflege \\
\hline Verhaltenstherapie \\
\hline European Child \& Adolescent Psychiatry \\
\hline Eastern Mediterranean Health Journal \\
\hline Ethiopian Journal Of Health Development \\
\hline Folia Phoniatrica Et Logopaedica \\
\hline European Addiction Research \\
\hline Australian Critical Care \\
\hline Contemporary Nurse \\
\hline Australian Journal Of Rural Health \\
\hline Australasian Psychiatry \\
\hline Annals Of Clinical Psychiatry \\
\hline Neuropsychology Review \\
\hline Journal Of Applied Sport Psychology \\
\hline International Psychogeriatrics \\
\hline American Journal Of Human Biology \\
\hline Gastroenterology Nursing \\
\hline Engineering Management Journal \\
\hline Journal Of Pediatric Oncology Nursing \\
\hline Journal Of Transcultural Nursing \\
\hline Epidemiology \\
\hline International Journal Of Human-Computer Interaction \\
\hline International Journal Of Methods In Psychiatric Research \\
\hline Neurorehabilitation \\
\hline
\end{tabular}




\begin{tabular}{|c|}
\hline Journal Of Adolescent Health \\
\hline Clinical Nursing Research \\
\hline Janac-Journal Of The Association Of Nurses In Aids Care \\
\hline Journal Of Nursing Care Quality \\
\hline Health Economics \\
\hline Psycho-Oncology \\
\hline American Journal Of Speech-Language Pathology \\
\hline Journal Of Science Education And Technology \\
\hline Adaptive Behavior \\
\hline Journal Of School Nursing \\
\hline Journal For Healthcare Quality \\
\hline Society \& Animals \\
\hline Quality Management In Health Care \\
\hline Journal Of Aging And Physical Activity \\
\hline Eating Disorders \\
\hline Experimental And Clinical Psychopharmacology \\
\hline Food And Drug Law Journal \\
\hline American Journal Of Geriatric Psychiatry \\
\hline Internet Research \\
\hline Harvard Review Of Psychiatry \\
\hline Journal Of The American Medical Informatics Association \\
\hline Journal Of Agricultural And Resource Economics \\
\hline Journal Of Engineering Education \\
\hline Structural Equation Modeling-A Multidisciplinary Journal \\
\hline Journal Of Wound Ostomy And Continence Nursing \\
\hline International Journal Of Human-Computer Studies \\
\hline Journal Of Law Medicine \& Ethics \\
\hline Neurobiology Of Learning And Memory \\
\hline Journal Of Family Nursing \\
\hline Psychiatric Services \\
\hline International Journal Of Occupational And Environmental Health \\
\hline Medical Care Research And Review \\
\hline Journal Of The American Psychiatric Nurses Association \\
\hline Journal Of Trauma Nursing \\
\hline Journals Of Gerontology Series A-Biological Sciences And Medical Sciences \\
\hline Journals Of Gerontology Series B-Psychological Sciences And Social Sciences \\
\hline Substance Use \& Misuse \\
\hline International Journal Of Electronic Commerce \\
\hline Journal Of Attention Disorders \\
\hline Aids Patient Care And Stds \\
\hline American Journal Of Managed Care \\
\hline Journal Of Addictions Nursing \\
\hline Journal Of Perianesthesia Nursing \\
\hline Evolution And Human Behavior \\
\hline Human Factors And Ergonomics In Manufacturing \& Service Industries \\
\hline
\end{tabular}




\begin{tabular}{|c|}
\hline Depression And Anxiety \\
\hline Families Systems \& Health \\
\hline Clinical Journal Of Oncology Nursing \\
\hline Journal Of Speech Language And Hearing Research \\
\hline Journal Of Behavioral Health Services \& Research \\
\hline Journal Of Ect \\
\hline Value In Health \\
\hline European Journal Of Public Health \\
\hline Scandinavian Journal Of Occupational Therapy \\
\hline Journal Of Forest Economics \\
\hline Chemistry Education Research And Practice \\
\hline Epidemiologia \& Prevenzione \\
\hline Anales Del Sistema Sanitario De Navarra \\
\hline Spanish Journal Of Psychology \\
\hline Pharmacoeconomics \\
\hline Journal Of Bioethical Inquiry \\
\hline Patient-Patient Centered Outcomes Research \\
\hline Journal Of Psychiatry \& Neuroscience \\
\hline Journal Of Agricultural \& Environmental Ethics \\
\hline Central European Journal Of Public Health \\
\hline International Journal Of Circumpolar Health \\
\hline Pratiques Psychologiques \\
\hline Turkish Journal Of Geriatrics-Turk Geriatri Dergisi \\
\hline Nursing Inquiry \\
\hline International Journal Of Nursing Practice \\
\hline Collegian \\
\hline Australian And New Zealand Journal Of Public Health \\
\hline Clinical Psychologist \\
\hline Kinesiology \\
\hline Environmental Health And Preventive Medicine \\
\hline Journal Of Psychiatric And Mental Health Nursing \\
\hline Natural Language Engineering \\
\hline Science And Engineering Ethics \\
\hline International Journal For Quality In Health Care \\
\hline Injury Prevention \\
\hline Health \& Place \\
\hline Cognitive Neuropsychiatry \\
\hline Psychology Health \& Medicine \\
\hline Ethnicity \& Health \\
\hline Journal Of Interprofessional Care \\
\hline Sport Education And Society \\
\hline Clinical Child Psychology And Psychiatry \\
\hline Aging \& Mental Health \\
\hline Transportation Research Part D-Transport And Environment \\
\hline Nursing In Critical Care \\
\hline
\end{tabular}




\begin{tabular}{|c|}
\hline Trends In Cognitive Sciences \\
\hline Australian Journal Of Agricultural And Resource Economics \\
\hline International Journal Of Geographical Information Science \\
\hline Transportation Research Part E-Logistics And Transportation Review \\
\hline Journal Of Child Health Care \\
\hline International Journal Of Language \& Communication Disorders \\
\hline Econometrics Journal \\
\hline Health Expectations \\
\hline Journal Of Clinical And Experimental Neuropsychology \\
\hline Computational And Mathematical Organization Theory \\
\hline Advances In Health Sciences Education \\
\hline Clinical Neuropsychologist \\
\hline Cognitive Systems Research \\
\hline Scandinavian Journal Of Public Health \\
\hline Physics In Perspective \\
\hline Hyle \\
\hline Cognition Technology \& Work \\
\hline Regional Environmental Change \\
\hline Zeitschrift Fur Psychosomatische Medizin Und Psychotherapie \\
\hline Australasian Journal On Ageing \\
\hline Nursing \& Health Sciences \\
\hline Geriatrics \& Gerontology International \\
\hline Rural And Remote Health \\
\hline International Journal Of Mental Health Nursing \\
\hline Sexual Health \\
\hline Australian Journal Of Primary Health \\
\hline Nicotine \& Tobacco Research \\
\hline European Journal Of Oncology Nursing \\
\hline New Genetics And Society \\
\hline Nursing Philosophy \\
\hline Online Information Review \\
\hline Psychology Of Sport And Exercise \\
\hline Quantitative Finance \\
\hline Law Probability \& Risk \\
\hline Journal Of Family Planning And Reproductive Health Care \\
\hline Bmc Geriatrics \\
\hline Nurse Education In Practice \\
\hline IMA Journal Of Management Mathematics \\
\hline Developing World Bioethics \\
\hline Bmc Palliative Care \\
\hline Bmc Womens Health \\
\hline Bmc Medical Education \\
\hline Bmc Medical Ethics \\
\hline Expert Review Of Pharmacoeconomics \& Outcomes Research \\
\hline European Journal Of Cardiovascular Nursing \\
\hline
\end{tabular}




\begin{tabular}{|c|}
\hline Child And Adolescent Mental Health \\
\hline International Journal Of Health Geographics \\
\hline Psychology And Psychotherapy-Theory Research And Practice \\
\hline Health And Quality Of Life Outcomes \\
\hline Revista Brasileira De Psiquiatria \\
\hline Revista Latino-Americana De Enfermagem \\
\hline Journal Of Hospice \& Palliative Nursing \\
\hline Current Psychiatry Reports \\
\hline M\&Som-Manufacturing \& Service Operations Management \\
\hline Pain Management Nursing \\
\hline American Journal Of Bioethics \\
\hline Nephrology Nursing Journal \\
\hline Journal Of Midwifery \& Womens Health \\
\hline Journal Of Nursing Scholarship \\
\hline Natural Hazards Review \\
\hline Advances In Skin \& Wound Care \\
\hline Stress And Health \\
\hline Advances In Neonatal Care \\
\hline Stata Journal \\
\hline Journal Of The Association For Information Systems \\
\hline Cin-Computers Informatics Nursing \\
\hline Traffic Injury Prevention \\
\hline Journal For Specialists In Pediatric Nursing \\
\hline Journal Of Womens Health \\
\hline Research And Theory For Nursing Practice \\
\hline Learning \& Behavior \\
\hline Annual Review Of Environment And Resources \\
\hline Worldviews On Evidence-Based Nursing \\
\hline Preventing Chronic Disease \\
\hline Journal Of Organizational And End User Computing \\
\hline Annual Review Of Clinical Psychology \\
\hline Journal Of Patient Safety \\
\hline Physical Review Special Topics-Physics Education Research \\
\hline JNP-Journal For Nurse Practitioners \\
\hline Medicc Review \\
\hline Journal Of Empirical Research On Human Research Ethics \\
\hline Journal Of Forensic Nursing \\
\hline International Journal Of Mental Health And Addiction \\
\hline Electronic Commerce Research And Applications \\
\hline International Journal Of Integrated Care \\
\hline Energy Efficiency \\
\hline Economics \& Human Biology \\
\hline Assistenza Infermieristica E Ricerca \\
\hline AJAR-African Journal Of AIDS Research \\
\hline South African Journal Of Psychiatry \\
\hline
\end{tabular}




\begin{tabular}{|c|}
\hline Universal Access In The Information Society \\
\hline Tobacco Induced Diseases \\
\hline Urban Forestry \& Urban Greening \\
\hline Global Health Action \\
\hline International Journal Of Environmental Research And Public Health \\
\hline International Journal Of Public Health \\
\hline Public Health Genomics \\
\hline Frontiers In Psychiatry \\
\hline Journal Of Nursing Research \\
\hline Ecology And Society \\
\hline World Psychiatry \\
\hline Acta Bioethica \\
\hline Psychiatry Investigation \\
\hline Journal Of Public Health \\
\hline Reproductive Health \\
\hline Japan Journal Of Nursing Science \\
\hline Journal Of Maps \\
\hline Annals Of General Psychiatry \\
\hline Globalization And Health \\
\hline Quarterly Journal Of Experimental Psychology \\
\hline Implementation Science \\
\hline Journal Of Neuropsychology \\
\hline Social Cognitive And Affective Neuroscience \\
\hline Journal Of Informetrics \\
\hline Early Intervention In Psychiatry \\
\hline Child And Adolescent Psychiatry And Mental Health \\
\hline International Journal Of Speech-Language Pathology \\
\hline Public Health Ethics \\
\hline International Emergency Nursing \\
\hline China Agricultural Economic Review \\
\hline Wiley Interdisciplinary Reviews-Climate Change \\
\hline Carbon Management \\
\hline Asia-Pacific Psychiatry \\
\hline Nature Climate Change \\
\hline Custos E Agronegocio On Line \\
\hline Health Information Management Journal \\
\hline Mathematics And Financial Economics \\
\hline Archaeological And Anthropological Sciences \\
\hline European Transport Research Review \\
\hline Translational Behavioral Medicine \\
\hline Nanoethics \\
\hline Women And Birth \\
\hline Neuroethics \\
\hline Biosemiotics \\
\hline Journal Of Mens Health \\
\hline
\end{tabular}




\begin{tabular}{|c|}
\hline Clinical Simulation In Nursing \\
\hline International Health \\
\hline Revista De Psiquiatria Y Salud Mental \\
\hline Journal Of Cancer Survivorship \\
\hline Disaster Medicine And Public Health Preparedness \\
\hline Disability And Health Journal \\
\hline Journal Of Studies On Alcohol And Drugs \\
\hline Engineering Studies \\
\hline IEEE Transactions On Learning Technologies \\
\hline Historical Studies In The Natural Sciences \\
\hline Autism Research \\
\hline Research In Gerontological Nursing \\
\hline Annual Review Of Resource Economics \\
\hline Transportation Letters-The International Journal Of Transportation Research \\
\hline Attention Perception \& Psychophysics \\
\hline SIAM Journal On Financial Mathematics \\
\hline Weather Climate And Society \\
\hline Water Alternatives-An Interdisciplinary Journal On Water Politics And Development \\
\hline Asian Nursing Research \\
\hline International Journal Of Design \\
\hline Journal Of Korean Academy Of Nursing \\
\hline Applied Economic Perspectives And Policy \\
\hline Bmj Quality \& Safety \\
\hline Epidemiology And Psychiatric Sciences \\
\hline Journal Of Global Health \\
\hline International Journal Of Nursing Knowledge \\
\hline Aslib Journal Of Information Management \\
\hline Journal Of Behavioral Addictions \\
\hline Sustainability \\
\hline Journal Of Sport And Health Science \\
\hline Workplace Health \& Safety \\
\hline Bariatric Surgical Practice And Patient Care \\
\hline Transportmetrica B-Transport Dynamics \\
\hline Jama Psychiatry \\
\hline History Of Geo- And Space Sciences \\
\hline Epj Data Science \\
\hline Journal Of Obsessive-Compulsive And Related Disorders \\
\hline Ecosystem Services \\
\hline Multisensory Research \\
\hline Lancet Global Health \\
\hline Lancet Psychiatry \\
\hline Iranian Journal Of Public Health \\
\hline Transportmetrica A-Transport Science \\
\hline Lgbt Health \\
\hline Language Cognition And Neuroscience \\
\hline
\end{tabular}


Journal of The American Association Of Nurse Practitioners

Journal Of The Association For Information Science And Technology

Physical Review Physics Education Research

Journal Of Professional Nursing

Developmental Neuropsychology 
Servicio de

Publicaciones

1542

Universidad Zaragoza 